\title{
A Survey on the Present State-of-the-Art of Explosives, Detection Methods and Automatic Explosive Detection using Wireless Sensor Network
}

\author{
R V Kishore Kumar ${ }^{1}$ \\ Research Scholar, Department of Computer Science and Engineering, \\ Acharya Nagarjuna University, Nagarjuna Nagar, Guntur District, Andhra Pradesh, India. \\ Dr. G.Murali \\ Professor, Research Guide, Department of Computer Science and Engineering, \\ Acharya Nagarjuna University, Nagarjuna Nagar, Guntur District, Andhra Pradesh, India.
}

\begin{abstract}
Though the There is ever increasing evidence of increased terrorist activities that spread entire world. In the wake of modern technologies and diversified threats, it is indispensable to comprehend, detect and prevent with reliable countermeasures. In this context detection of explosives is an active research area. Towards this end many detection systems came into existence. The government agencies in various countries are equipped with the devices that can detect explosives. However, often they are bulky in nature and they are publicly visible and expensive. Therefore it is not feasible to use them in the present condition in public places such as railway stations, airports, bus stations and so on. Manual intervention is another problem in the traditional systems. Therefore there is immediate need for the detection of explosives to be incorporated in a mobile network like Wireless Sensor Network (WSN). Since the nodes of WSN can be deployed hidden from public view, such network is an ideal candidate to have real time and automated detection of explosives. The realization of such networks can pave the way for installation at public domains to safeguard lives and property of citizens. Towards this end we review the literature on the present state-of-the-art of explosives, their characterization, detection methods including the usage of Wireless Sensor Networks (WSNs) for automatic and real time explosive detection. This paper gives insights into different insights into explosives and detection methods that can help in further research towards realizing a fool proof system for explosive detection.
\end{abstract}

Keywords: Explosives, explosive detection methods, WSN, characterization of explosives

\section{Introduction}

There is lot of attention in the research area pertaining to detection of explosives in the wake of terror attacks across the globe. The world has sufficient evidence of this violence and several thousands of people lost their lives and even more number of people injured. Government infrastructures and common people are targets to terrorists. Improvised Explosive Devices (IEDs) are used by anti-social elements for making terror attacks from a remote place using mobile phones and other devices. Therefore it is essential to have reliable detection systems to protect people and property from dangerous attacks. Conventional systems used to detect explosives are expensive and bulky in nature. Moreover they need manual attention besides being visible publicly which causes intruders to change their plans accordingly. Building an automated detection network which can be deployed easily and the network is not physically visible is very important concern. In other words the network to detect explosives can be hidden from view of public so that it does its job automatically to detect explosives and intimate the people concerned. However, building such robust system is very challenging. Of late some attempts were found in the literature to safeguard interests of public and property by deploying sensors to form wireless sensor network which can detect IEDs and the chemicals used in the materials. However, this research can be considered to be in its infancy and there is much room for research into the area of detecting explosives using wireless sensor network. In this research we explore the ways and means to propose a mechanism and implement it by taking help of possible inter-disciplinary study and usage of required equipment. From the initial study it is conceived that the proposed system consists of a processor, sensor and wireless transceiver equipment. The system will be able to collect sensor data and perform processing of data using the proposed methodology and notifies security officials about any suspicious material. Our contribution in this paper includes the review of literature to provide insights into different explosives and their detection methods. We intend to propose a mechanism for WSN to detect explosives. This paper is structured as follows. Section 2 throws light into wireless sensor networks, their applications and challenges. Section 3 focuses on detection of explosives in soil and water. Section 4 gives details about WSN and sensor networks for detection of explosives. Section 5 throws light into commercially available explosive detection technologies. Section 6 provides Ion Mobility Spectrometry for examining organic explosives. Section 7 provides more information on explosives detection categories and methods. Section 8 focuses on the characterization of explosives for detection methods. Section 9 provides material discrimination approach for explosives detection while section 10 concludes the paper besides providing directions for future work. 


\section{Wireless Sensor Networks Applications and Challenges}

Sensor networks can offer sensing, communication and computing capabilities. The usage of such network became ubiquitous. The applications are in many diverse fields like education, studying wildlife habitat, surveillance, agriculture, micro-surgery, child education and military to mention few. Stringent energy constraints throw many challenges in WSNs [1], [15], [17], [27], [33]. The reason behind this is that sensor nodes operate in unattended environments with battery power [2], [46]. There are some other applications of WSN such as geophysical monitoring, precision agriculture, tracking animal herds, traffic monitoring, and supply chain management. The characteristics of WSN include subjected to failures, limited energy, node mobility, heterogeneity, communication failures, scalability issues, hostile environment, and so on [3], [4]. Clustering algorithms can be used in WSN for different security applications [12], [51]. Using deployment knowledge, key management schemes came into existence in WSN for secure communications [13]. When WSN is made up of thousands of nodes, a real time communication needs robust architecture as discussed in [14]. The location aware nodes in WSN can participate in security applications with ease [16]. Clock synchronization is one of the methods for efficiency in WSN as temporal events are to be identified [18], [49]. Low power wireless sensor networks not only vulnerable but they are influenced by resources [19]. Distributed localization also helps in WSN for better and accurate performance in target tracking and other applications [20], [21], [31]. WSNs work with different routing protocols and they support distributed routing with desired Quality of Service (Qos) [22], [44]. More research on QoS of WSN can be found in [38]. Heterogeneity, energy efficiency and clustering concepts of WSN help to use it for detection of explosives experiments [23], [39], [42]. Secure routing in presence of attacks explored in [40].

Networking issues in WSN can help to characterize it for detecting explosives. The issues pertaining to networking can be found in [24]. Communication procedures in WSN often provide discrepancies between the simulations and ground truth [25]. Wireless sensor networks can be deployed in different ways including hierarchical where groups of nodes perform different tasks [26]. WSNs support different MAC protocols such as S-MAC, WiseMAC, Traffic-Adaptive MAC, SIFT, DMAC, and Timeout - MAC. There are multimedia sensors that can help in visualizing detection of explosives. Such useful information can be found in [30]. Mobile relay configuration is explored in [34] for ensuring the huge data can be transmitted in mobile networks efficiently. Radio Irregularity Model (RIM) in WSN is explored in [36] which can help while the network is employed for explosive detection. Energy efficient surveillance system can be made using WSN [37]. This kind of capability of WSN can be used to build explosive detection capabilities. Self organization is also possible with WSN [41]. Distributed signal processing in WSN can have its effect on scalability, bandwidth efficiency, and robustness [43]. Elliptic Curve Cryptography is one of the approaches to secure communication networks. Liu \& Ning [45] explored configurable library for ECC. Sensor nodes can work in collaboration with actor nodes as discussed in [47]. Target tracking is one of the important features of WSN that can also be explored in detection of explosives context. The nodes in WSN can form into clusters for effective and acoustic target tracking [50]. WSN is being used for habitat monitoring [52]. This feature can be extended to monitor surroundings and even detect explosives.

\section{Detection of Explosives in Soil And Water}

Explosives are kept in soil and water in many countries. They were under control of respective governments. However, maintaining such sites and monitoring them besides detecting them play a pivotal role in the maintenance of those sites. Towards detection of explosives, electronic sensors can participate. Watson \& McGuire [4] explored Surface Acoustic Wave (SAW) and Electronic Nose for detection of explosives. Their research was carried out in the context of explosives dumped by USA government for military purposes. They provide details of Model 7100 Vapor Analysis System for quantifying vapors and thus detect explosives. Identification of different material like 2,6dinitrotoluene, 2,4-dinitrotoluene, hexahydro-1,3,5-trinitro1,3,5-triazine (RDX), and 2,4,6-dinitrotoluene were used in the experiments. SAW crystals are widely used for experiments on detection of explosives. SAW crystals are exposed to different polymer coatings pertaining to vapour to be characterized. Reduction in sensitivity is observed with polymer coatings to SAW crystals. To detect explosives different methods were used. They are known as calibration, soil analysis, and water analysis. The results revealed that the performance of the methodology used for experiments could be improved further. This is because the results of experiments are not matching with the ground truth.

\section{Sensors or WSN to Detect Explosives}

Sundram [5], a major in Singapore armed forces, conducted experiments with WSN for explosive detection. The main focus of his research was on Improvised Explosive Devices (IEDs) as part of surveillance in public places. Infrared and magnetic sensors were used for experiments. The environment used to have experiments is a public mall and the street nearby. The threat model contains IED emplacement. A collection of sensors were built and positioned in the environment. Ferromagnetic materials are used as explosives. Human subjects were asked to carry explosive materials and proceed towards receptacle. Two types of detectors were used in sensors. They are known magnetic detectors and infrared detectors. Magnetic sensors were able to detect the explosives while the infrared sensors could not do it correctly due to background infrared radiation. As the results reveal, this technology can be used to protect communal areas and public places such as airports. Bieleckiet al. [10] explored how sensors and systems can be used that can be used to detect explosive devices. Improvised Explosive Devices (IEDs) and counter measures are the important contents in their research. Sensors are the devices that can sense environment to capture data and analyze. In the process the sensor devices can be programmed for 
detection of explosives. For instance sensors can be of chemical sensors, electrochemical sensors, chromatography sensors and so on. Based on the kind of sensing work they do they are known. Explosives are more than hundred in number. However, they have certain common characteristics such as geometry, material density, elemental composition, and vapor emissions. The geometry can be analyzed using image shape analysis. The material density reflects the probability of explosives since explosives are denser than other materials. Elemental composition can be analyzed using vapor emission analysis. Vapor emissions can be using vapor samples and analyzing them [10]. The vapor concentration of explosives can be categorized into three categories such as A, B and C based on the concentration of air at room temperature.

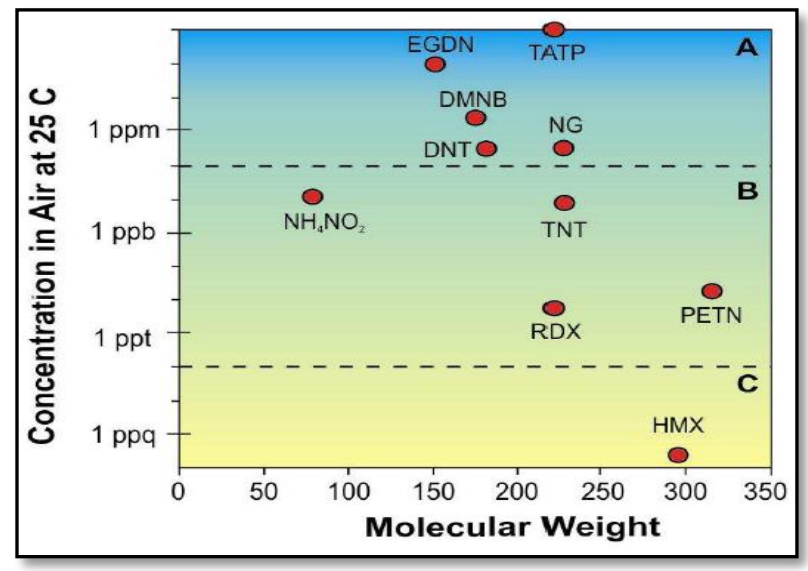

Figure 1: Identification of explosives based on molecule weight and vapor concentration [10]

As can be seen in Figure 1, it is evident that the molecule weight and concentration of air at room temperature it is possible to determine the explosives. The detection techniques such as bulk or trace detection methods can be employed in order to identify explosives with certain probability. Based on these factors various sensor devices are manufactured that can help in detecting explosives [10]. As the sensors in the real world might be bulky and cannot be installed without public visibility, it is essential to find alternatives to the traditional devices. In our future research we intend to build sensing capabilities into nodes in a WSN that can be installed and used with ease without revealing its presence to public. Automatic detection of explosives is the highly desirable research in the real world as of now. In the wake of terrorist attacks and the alarming nature of their activities and projected damage, it is essential to have successful networks that gather counter intelligence and take necessary steps. An attempt has been made in [28] towards automatic detection of explosives using WSN. Detection architecture was proposed as shown in Figure 2.

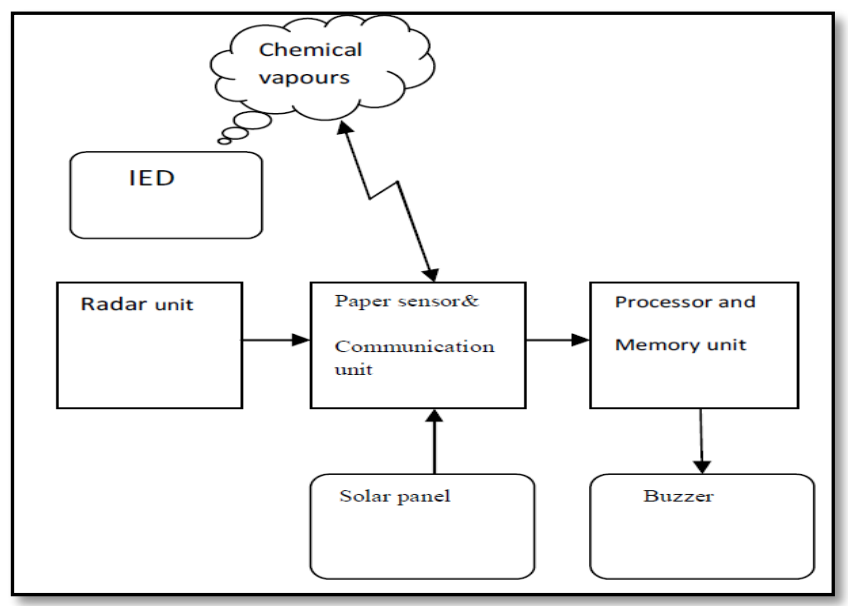

Figure 2: Explosive detection architecture [28]

As can be seen in Figure 2, it is evident that IED's chemical vapors are used as inputs to the system. Radar unit, paper sensor, communication unit, processor and memory unit, buzzer, solar panel are used in the architecture. The solar panel module is used to have power source. Paper sensor is used to trace the vapors of different explosives used in the experiments. The communication unit is responsible to have communication with other sensors in the network. Processor plays a vital role in processing the sensed data. To track LED movement, the radar unit is used. The buzzer is used to indicate the explosives when matched correctly. This kind of experimentation in the real world is expensive. In our future work, we intend to make this kind of experiment with simulations. Simulation study can prove the concept before realizing the technique in the real world.

\section{Commercially Available Explosive Detection Technologies}

Thiesanet al. [6] made a review of commercially available explosive detection technologies. Explosive detection methods are classified into two types. They are trace detection of vapor/particles and bulk detection. The bulk detection methods are of two types. They are imaging methods and nuclear-based methods.

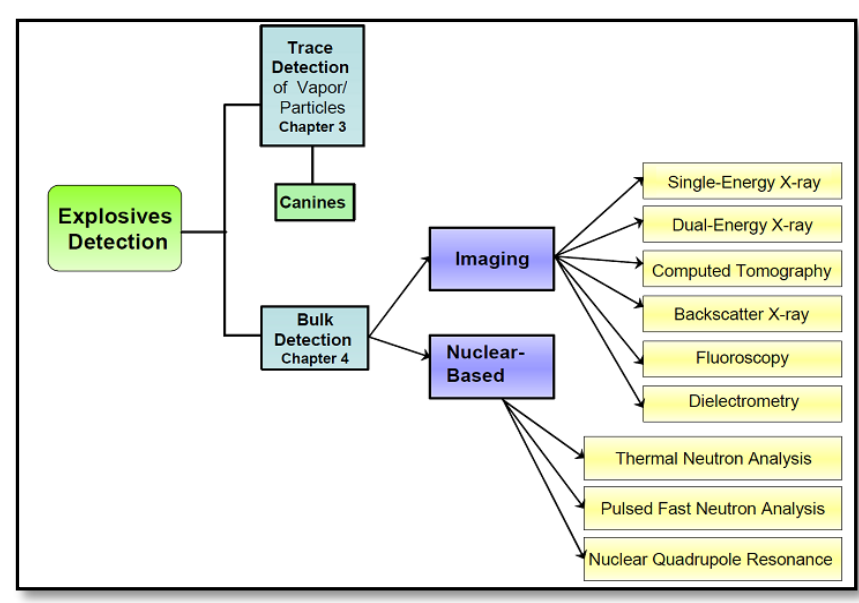

Figure 3: Explosive detection technologies (excerpt from [6]) 
The imaging kind of bulk detection technologies are singleenergy X-ray, dual-energy X-ray, computed tomography, backscatter X-ray, Fluoroscopy, and Dielectrometry. The nuclear based methods include thermal neutron analysis, pulsed fast neutron analysis, nuclear quadrupole resonance. There are many advantages associated with explosives detection. Some of the potential applications include screening of personnel on the fly, screening of large number of vehicles, and shipped items. They are also used for bomb search, handle special situations and protection of special infrastructure. However the screening techniques throw health issues that are to be considered.

\section{Ion Mobility Spectrometry for Examining Organic Explosives}

Koyuncu et al. [7] studied organic explosives using Ion Mobility Spectrometry (IMS). They proposed an analytical method and implemented it. The method has three phases. In the first phase study of standard mixture solutions of TETRYL, PETN, RDX and TNT is made using IMS. In the second phase, real explosive residues are used to have qualitative analysis. In the third phase experiments were carried out to trace explosive residues. IMS also known as plasma chromatography is one of the analytical methods that can separate ionized gas molecules based on the mobility. An IMS system needs suitable ion source to function well. A beta emitter is used typically as ion source. Reactant ions are predominantly used in the experiments. The reactant ions and molecules are involved in the process of studying the possible dynamics and characteristics of explosives. Post blast residue samples were used for the experiments where organic explosives are studied using IMS system. The IMS can be carried out with less sample volume when compared with other methods.

\section{Explosive Detection Categories and Methods}

David and Lewis [8] explored different explosive detection techniques and their categories. Especially those techniques are used for protecting borders, airports and so on. Trace and Bulk are two major categories of explosive detection methods. Trace detection refers to the detection method that makes use of small quantities of explosives. These explosives are in two forms such as vapor or particulate. The former is in the air while the latter is the residue of explosive material that adheres to surfaces of the subject premise. Bulk explosive detection methods can detect macroscopic masses of explosives that are visible to human eye and they have high penetrating capacity for maximum destruction.

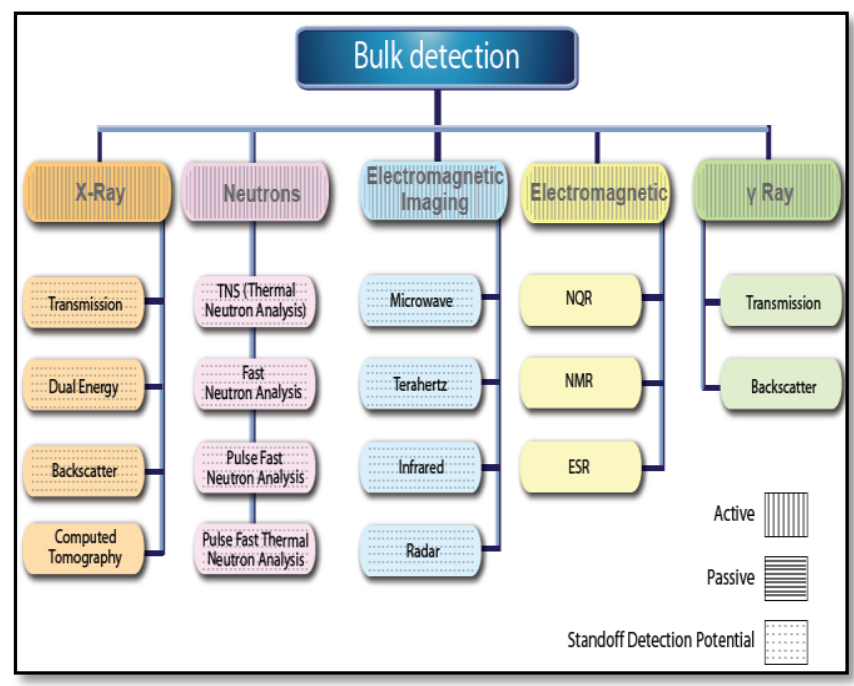

Figure 4: Bulk detection techniques of different kinds [8]

As presented in Figure 4, it is evident that there are five categories of bulk detection methods. Again in each category there are methods with active, passive and standoff detection potential. The categories are X-Ray, Neutrons, Electromagnetic Imaging, Electromagnetic and Y Ray. The $\mathrm{X}$-Ray methods are based on transmission, dual energy, backscatter, and computed tomography. The neutrons methods include thermal, fast, pulse fast and pulse fast thermal neutron analysis. The electromagnetic imaging methods include microwave, terahertz, infrared, and radar. The electromagnetic include NQR, NMR, and ESR while the $Y$ Ray techniques are based on transmission and backscatter [8].

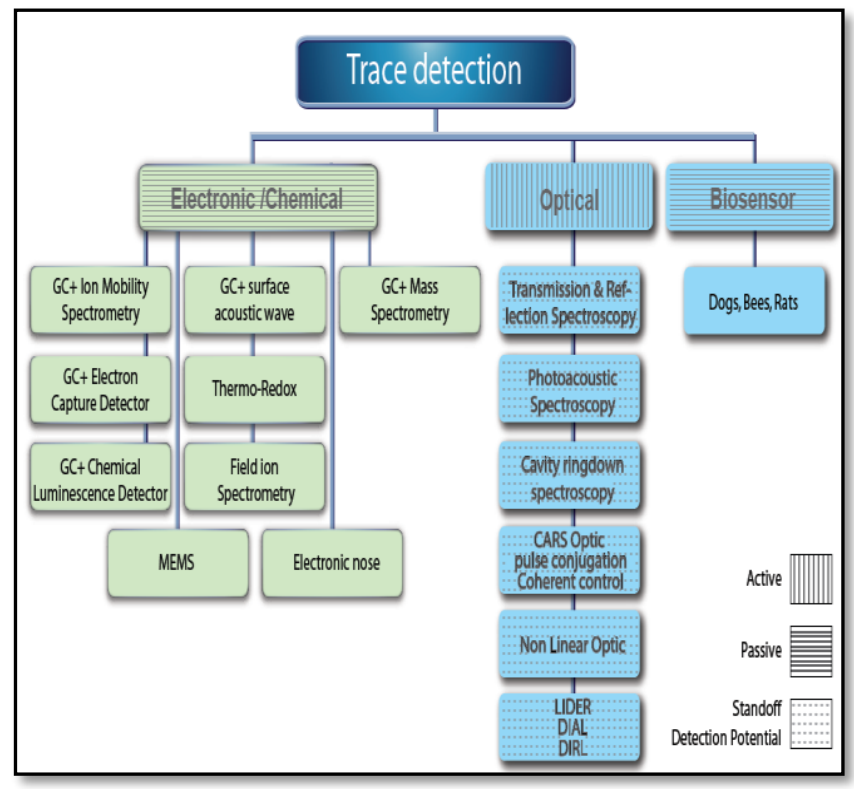

Figure 5: Trace detection techniques [8]

As explored in [8] the trace detection methods are categorized into Electronic/Chemical, Optical and Biosensor. There are many methods pertaining to electronic/chemical category such as GC+ Ion Mobility Spectrometry, GC+ surface acoustic wave, GC+ Mass Spectrometry, GC+ 
Electron Capture Detector, Thermo-Redox, GC+ Chemical Luminescence Detector, Field, Ion Spectrometry, MEMS and Electronic Nose. The optical category includes Transmission \& Reflection Spectroscopy, Photo acoustic Spectroscope, Cavity Ringdown Spectroscopy, CARS Optic pulse conjugation coherent control, non linear optic, LLIDER, DIAL, and DIRL. The Biosensor methods are based on Dogs, Bees, and Rats. These methods are identified into three different types such as active, passive and standoff detection potential [8]. As discussed in [9] Home Made Explosives (HMEs) are being used by terrorists and the detection methods need to be tailored to meet this requirement. In fact, many attacks on USA revealed the fact.

\section{Characterization of Explosives For Detection Methods}

As a matter of fact, it is an essential to characterize explosives prior to detection. Since characterization can help programs to learn features of explosives, it is imperative to do so. The characterization can help researchers to automate the detection process. We believe that the characterization can help in developing and deploying WSN for the purpose of explosive detection intuitively. Tourne [11] explored forensic research for characterization and detection of explosives. In order to characterize explosives different kinds of properties are to be studied and analyzed. Therefore the characterization can be done in terms of electromagnetic, thermal, acoustic, mechanical, physical and chemical properties. The material of explosives has to be subjected to interrogation and analysis. Successful characterization of explosive substances can help improve the accuracy of detection.Traces of IEDs can be used for mechanical characterization. The mechanical characterization of explosives is based on the traces that adhere to surfaces. The explosions done for military purposes can be used to collect traces and the mechanical characterization is based on the adherence of traces to other components. The roughness and the particle - substrate orientations are used for mechanical characterization. The physical characterization is pertaining to detonation and shock sensitivity. There are many properties related to density, crystal morphology, particle size and surface area. Especially morphology data plays a vital role in physical characterization [11]. With respect to thermal characterization, mechanical and thermal excitations play a big role. The vapor pressures of explosives are sensitive to temperatures. This fact provides needful insights to find origin of induced chemical decomposition of explosives. Acoustic characterization refers to the damage evolution. The damage determination is studied using computed tomography (CT), and acoustic emission. Electromagnetic characterization is pertaining to terahertz $(\mathrm{THz})$ responses. Based on $\mathrm{THz}$, it is possible to characterize explosives. The quantitative analysis of electromagnetic responses can provide required insights to characterize such explosives. The most advanced detection techniques that exploit these characterizations include Mass Spectrometry, Ion Mobility Spectrometry, Terahertz Spectroscopy, Infrared Spectroscopy, Laser induced breakdown spectroscopy, raman spectroscopy, and surface-enhanced raman spectroscopy. In the process of detecting explosives sensor technologies play a vital role. There are many sensors such as SAW sensors, QCM sensors and MEMS sensors. Carbon nanotube-based sensors were also investigated to exhibit detection of explosives [11].

\section{Material Discrimination Approch For Explosives Detection}

Explosives are generally carried in luggage bags rucksacks or such carriers. Therefore one of the important technologies to detect such material is X-ray. Stated differently X-ray Computed Tomography (CT) is the best known way to reach materials that are kept in bags and other containers. Thus the inside things can be understood programmatically. Then Multi-Energy CT (MECT) can be used in order to gain more details about the material being studied. In the machine learning perspective, the detection of explosives using MECT can be done using the architecture presented in [32].

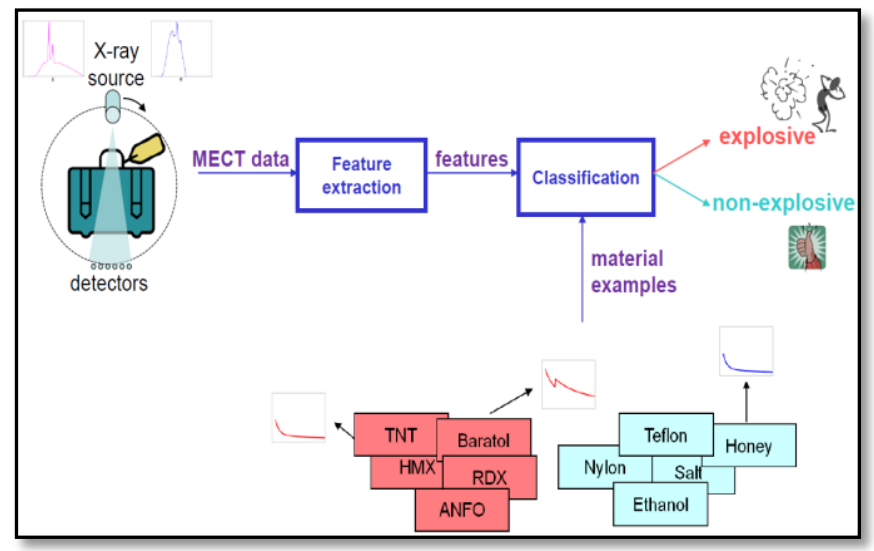

Figure 6 : Machine learning perspective for explosives detection [32]

As can be seen in Figure 6, it is evident that the material samples are taken into a bag which is subjected to X-ray source. Later, as discussed earlier, MECT is used to extract more details and MECT data is sent for feature extraction. The extracted features are classified into explosives and nonexplosives. Conventional mechanisms might not be able to detect accurately. However, with emerging technologies in the field of MECT can be used to improve detection accuracy. Since the MECT extracts more features that can help in increasing the scope of detection. Learning and using features adaptively can help increase the detection performance. However, the detection results are ultimately limited by the fundamental limitations of MECT technology [32]. In our future work, we intend to explore possible means of improving detection performance to eliminate false alarms and increase throughput.

X-rays technology has been around for explosive detection. Rebuffel et al. [35] explored more on it. X-ray technologies that are based on dual-energy radiography can provide characterization at broader level. Further improvement is made with semi-conductors based X-ray detectors. They explored different Explosive Detection Systems (EDSs) that are based on X-ray. CdTe/CdZnTe detectors were proved to be better than their counterparts like Germanium detectors. For some specific configurations, the alternative technique 
proved to be useful is Backscatter. Local precise characterization was made possible with spectrometric detectors. Out of all the detectors $\mathrm{CdTe} / \mathrm{CdZnTe}$ are well adapted ones. As explored in [53] complex biological samples can be studied and discriminated by using different techniques such as Ion Mobility - Mass Spectometry for the purpose of proteomics. It can be used to probe into biophysical features towards detection of explosives.

\section{Conclusions and Future Work}

Man has made tremendous progress in scientific inventions by exploiting natural laws. However, the ever-increasing threat of terrorism became a real barrier to the development of nations across the globe. Terrorists have been using explosives to disrupt normalcy in countries intentionally. Sometimes, the counter measures proved to be ineffective due to the apaptation of new mechanisms or technologies. Therefore it is inevitable to march towards the research that can provide fool proof mechanisms to protect property and life of public. Towards this end, one of the approaches is to have ability to detect explosives early and automatically. The present detection devices are bulky and their presence is known to anyone. This helps anti-social elements to change their plans accordingly. An important insight into this is to have a network like WSN for detecting explosives automatically. Since the sensor nodes used in WSN are managed to be hidden from pubic view, this network is more efficient when there is perfect methodology that can be used in sensor nodes for automatic detection of explosives. We intend to build such methodology in future with simulation study. Prior to that we felt it was useful to have the review of the present state-of-the-art of explosives, their detection methods and identified the need for proposed a mechanism for WSN to detect explosives in the real time. Towards this end, in this paper, we threw light into different aspects of explosives, their characterization, detection and possible technologies for counter measures.

\section{References}

[1] Daniele Puccinelli and Martin Haenggi. (2005). Wireless Sensor Networks: Applications and Challenges of Ubiquitous Sensing. IEEE. p.12-17.

[2] Youngmin Kim, Hyojeong Shin, and Hojung Cha. (2008). Y-MAC: An Energy-efficient Multi-channel MAC Protocol for Dense Wireless Sensor Networks. IEEE. p.25-34.

[3] Kay romar,rahul.p. (2008). middl chanllege wsn. IEEE. p.213-313.

[4] Gary W. Watson and David S. McGuire, Detection Of Explosives In Soil And Waterwith An Electronic Nose, 1999, p1-10.

[5] Joshua Sundram and Phua Poh Sim, Using Wireless Sensor Networks In Improvised Explosive Device Detection, 2007, p1-91.

[6] Lisa Thiesan, David Hannum, Dale W. Murray, John E. Parmeter, Survey of Commercially Available Explosives Detection Technologies and Equipment 2004, Sandia National Laboratories, 2005, p1-97.
[7] H"ulya KOYUNCU, Ertan SEVEN and Ayla CALIMLI, Examination of Some Organic Explosives by IonMobility Spectrometry (IMS), 2005, p255-264.

[8] J David and A M Lewis, Explosive Detection Equipment and Technology for Border Security, JRC scientific and technical reports, 2008, p1-62.

[9] Dr. Ruth Doherty, Detection of Homemade Explosives (HMEs), 2009, p1-4.

[10] ZbigniewBielecki, Jacek Janucki, Adam Kawalec, Janusz Mikołajczyk, Norbert Pałka, Mateusz Pasternak, Tadeusz Pustelny, Tadeusz Stacewicz, Jacek Wojtas, Sensors And Systems For The Detection Of Explosive Devices -An Overview, 2012, p3-28.

[11] Marilyn Tourné, Developments in Explosives Characterization and Detection, Forensic Research, 2013, p1-10.

[12] Ameer Ahmed Abbasi a, Mohamed Younis . (2007). A survey on clustering algorithms for wireless sensor networks. ELsevier. 30 (1), p.32-44.

[13] Wenliang Du,Jing Deng. (2004). A Key Management Scheme for Wireless Sensor Networks Using Deployment Knowledge. IEEE. p.56-60.

[14] Chenyang Lu Brian M. Blum Tarek F. Abdelzaher John A. Stankovic Tian He. (2008). RAP: A RealTime Communication Architecture for Large-Scale Wireless Sensor Networks. IEEE. p.25-34.

[15] I.F. Akyildiz, W. Su Y. Sankarasubramaniam, E. Cayirci. (2002). Wireless sensor networks: a survey. ELsevier. 38 (1), p.213-313.

[16] Rong Peng and Mihail L. Sichitiu. (2006). Angle of Arrival Localization for Wireless Sensor Networks. ELsevier. p.12-17.

[17] Th. Arampatzis, J. Lygeros. (2005). A Survey of Applications of Wireless Sensors and Wireless Sensor Networks. IEEE. p.23-33.

[18] Bharath Sundararaman, Ugo Buy, and Ajay D. Kshemkalyani. (2005). Clock Synchronization for Wireless Sensor Networks: A Survey. IEEE.p.213313.

[19] Deepak Ganesan y , Deborah Estrin. (2008). Complex Behavior at Scale: An Experimental Study of LowPower Wireless Sensor Networks.IEEE.p.12-17.

[20] Koen Langendoen , Niels Reijers. (2003). Distributed localization in wireless sensor networks: a quantitative comparison. ELsevier. 43 (1), p.25-34

[21] Mihail L. Sichitiu and Vaidyanathan Ramadurai. (2003). Localization of Wireless Sensor Networks with a Mobile Beacon. ELsevier.p.23-33.

[22] Ze Li,Haiying Shen. (2014). A QoS-Oriented Distributed Routing Protocol for Hybrid Wireless Networks. ELsevier. 13 (4), p.25-34.

[23] Li Qing, Qingxin Zhu, Mingwen Wang. (2006). Design of a distributed energy-efficient clustering algorithm for heterogeneous wireless sensor networks. ELsevier. 29 (1), p.2230-2237.

[24] Ye, Wei Yu, Yan Zhao, Yonggang Estrin, D. (2006). Networking Issues in Wireless Sensor Networks. CENS.p.23-33. 
[25] Wong, J L Kuang, Louane Potkonjak. (2005). Statistical Model of Lossy Links in Wireless Sensor Networks. CENS. p.23-33.

[26] Jason Hill, Mike Horton, Ralph Kling. (2004). THE PLATFORMS ENABLING WIRELESS SENSOR NETWORKS. CENS. 47 (6), p.23-33.

[27] E. Ilker Oyman and Cem Ersoy. (1997). Multiple Sink Network Design Problem in Large Scale Wireless Sensor Networks. IEEE.p.213-313.

[28] Avinash.Vanimireddy and D.Aruna Kumari, Detection of Explosives Using Wireless Sensor Networks, International Journal of Engineering Trends and Technology- Volume3Issue3- 2012, p1-4.

[29] Ilker Demirkol, Cem Ersoy, and Fatih Alagöz. (2012). MAC Protocols for Wireless Sensor Networks: a Survey. ELsevier. p.25-34.

[30] Ian F. Akyildiz , Tommaso Melodia, Kaushik R. Chowdhury. (2007). A survey on wireless multimedia sensor networks. ELsevier. 51 (1), p.921-960.

[31] Loukas Lazos and Radha Poovendran. (2004). SeRLoc: Secure Range-Independent Localization for Wireless Sensor Networks. IEEE. p.213-313.

[32] Limor Eger PrakashIshwar, W. Clem Karl, Homer Pie, Material Discrimination Using Multi-Energy XRay Computed Tomography for Explosives Detection, p1-10.

[33] Aleksandar Milenkovic, Chris Otto, Emil Jovanov. (2006). Wireless sensor networks for personal health monitoring: Issues and an implementation. ELsevier. p.12-17.

[34] Fatme El-Moukaddem, Eric Torng, and Guoliang Xing. (2013). Mobile Relay Configuration in DataIntensive Wireless Sensor Networks. IEEE. 12 (2), p. $25-34$.

[35] Veronique REBUFFEL, Jean RINKEL, Joachim TABARY, Loick VERGER CEA-LETI, New Perspectives of X-ray Techniques for Explosive Detection Based on CdTe/CdZnTe Spectrometric Detectors, International Symposium on Digital Industrial Radiology and Computed Tomography, p18.

[36] Gang Zhou, Tian He, Sudha Krishnamurthy, John A. Stankovic. (2004). Impact of Radio Irregularity on Wireless Sensor Networks. IEEE. p.12-17.

[37] Tian He, Sudha Krishnamurthy, John A. Stankovic, Tarek Abdelzaher, Liqian Luo, Radu Stoleru, Ting Yan, Lin Gu. (2004). Energy-Efficient Surveillance System Using Wireless Sensor Networks. IEEE.p.213313.

[38] Dazhi Chen and Pramod K. Varshney. (2004). QoS Support in Wireless Sensor Networks: A Survey. IEEE. p.32-44.

[39] Karim Seada, Marco Zuniga, Ahmed Helmy, Bhaskar Krishnamachari. (2004). EnergyEfficient Forwarding Strategies for Geographic Routing in Lossy Wireless Sensor Networks. ACM. p.56-60.

[40] Chris Karlof, David Wagner. (2013). Secure routing in wireless sensor networks: attacks and countermeasures. ELsevier. 1 (1), p.293-315.
[41] Katayoun Sohrabi, Jay Gao, Vishal Ailawadhi and Gregory J Pottie. (1999). Protocols for SelfOrganization of a Wireless Sensor Network.IEEE. p.12-17.

[42] Joseph Polastre,Jason Hill,David Culler. (2004). Versatile Low Power Media Access for Wireless Sensor Networks. ACM.p.23-33.

[43] In-Jun Xiao, Alejandro Ribeiro, Zhi-Quan Luo, and Georgios B. Giannakis. (2006). Distributed Compression-Estimation Using Wireless Sensor Networks. ACM. p.213-313.

[44] Kemal Akkaya, Mohamed Younis. (2005). A survey on routing protocols for wireless sensor networks. ELsevier. 3 (2), p.325-349.

[45] An Liu, Peng Ning. (2004). TinyECC: A Configurable Library for Elliptic Curve Cryptography in Wireless Sensor Networks. deement of science. p.32-44.

[46] Alejandro Ribeiro. (2006). Bandwidth-Constrained Distributed Estimation for Wireless Sensor Networks-Part II: Unknown Probability Density Function. IEEE. p.25-34.

[47] Ian F. Akyildiz, Ismail H. Kasimoglu. (2004). Wireless sensor and actorq networks: research challenges. ELsevier. 2 (1), p.351-367.

[48] Mark A. Perillo and Wendi B. Heinzelman, Wireless Sensor Network Protocols, p1-35.

[49] Jeremy Elson. (2002). Wireless Sensor Networks: A New Regime for Time Synchronization. ACM. p.5660.

[50] Wei-Peng Chen,Jennifer C. Hou. (2004). Dynamic Clustering for Acoustic Target Tracking in Wireless Sensor Networks. . 3 (3), p.12-17.

[51] Paolo Baronti b,c, Prashant Pillai a, Vince W.C. Chook a, Stefano Chessa b, Alberto Gotta b, Y. Fun $\mathrm{Hu}$. (2007). Wireless sensor networks: A survey on the state of the art and the 802.15.4 and ZigBee standards. ELsevier. 30 (1), p.1655-1695.

[52] Alan Mainwaring1 Joseph Polastre2 Robert Szewczyk David Culler John Anderson. (2002). Wireless Sensor Networks for Habitat Monitoring.ACM. p.25-34.

[53] John A. McLean, Brandon T. Ruotolo, Kent J. Gillig, David H. Russell, Ion mobility-mass spectrometry: a new paradigm for proteomics, Elsevier, International Journal of Mass Spectrometry 240 (2005) p301-315. 